\title{
Philosophiques
}

\section{Précis de Reconstructing Reason and Representation}

\section{Murray Clarke}

Volume 34, numéro 2, automne 2007

URI : https://id.erudit.org/iderudit/016997ar

DOI : https://doi.org/10.7202/016997ar

Aller au sommaire du numéro

Éditeur(s)

Société de philosophie du Québec

ISSN

0316-2923 (imprimé)

1492-1391 (numérique)

Découvrir la revue

Citer ce document

Clarke, M. (2007). Précis de Reconstructing Reason and Representation.

Philosophiques, 34(2), 353-362. https://doi.org/10.7202/016997ar

Ce document est protégé par la loi sur le droit d'auteur. L'utilisation des services d'Érudit (y compris la reproduction) est assujettie à sa politique d'utilisation que vous pouvez consulter en ligne.

https://apropos.erudit.org/fr/usagers/politique-dutilisation/ 


\title{
Précis de Reconstructing Reason and Representation
}

\author{
MURRAY CLARKE \\ Université Concordia
}

J'avais deux objectifs à l'esprit quand j'ai écrit Reconstructing Reason and Representation. Premièrement, j'ai voulu clarifier et évaluer les mérites empiriques et conceptuels de la psychologie évolutionniste. Deuxièmement, j'ai voulu évaluer les implications de la psychologie évolutionniste pour certains thèmes de l'épistémologie, la philosophie des sciences et la philosophie de l'esprit. Je voulais offrir, durant le processus, une nouvelle approche de l'épistémologie naturaliste. L'idée était que nous devions permettre à l'épistémologie de devenir modulaire et la percevoir comme un ensemble de genres naturels. Ma conviction était qu'après avoir fait ce changement nous pourrions libérer l'épistémologie de la stagnation scolastique de l'analyse conceptuelle. J'ai essayé de poser les bases pour ce projet en élucidant les idées centrales qui constituent la version de la psychologie évolutionniste de Leda Cosmides et John Tooby comme suit. Cosmides et Tooby ont adopté l'affirmation méthodologique de la cohérence trans-théorique ou, comme l'appelle Jerry Fodor: «la pertinence explicative réciproque ». Par exemple, la biologie de l'évolution et la psychologie cognitive se contraignent mutuellement pour l'acceptabilité des théories que chacune peut intégrer. Elles souscrivent aussi à l'idée de nature humaine universelle quant aux mécanismes psychologiques complexes, ou modules darwiniens, et non quant à l'expression des comportements culturels. Deuxièmement, ces mécanismes psychologiques complexes sont des adaptations qui ont été élaborées par la sélection naturelle au cours d'une certaine période évolutionnaire. Par conséquent, les modules darwiniens sont des structures cognitives innées dont les propriétés principales sont largement déterminées par des facteurs génétiques. Une troisième idée est que la structure complexe de l'esprit humain est adaptée au mode de vie des chasseurs-cueilleurs du Pléistocène, et pas nécessairement à nos circonstances modernes. Ce que nous pensons de l'histoire humaine récente, c.-à-d. les deux derniers millénaires, n'a pas grand-chose à voir avec la formation de l'esprit humain, parce que nos esprits ont été largement formés par les deux derniers millions d'années comme chasseurs-cueilleurs du Pléistocène. Une quatrième idée est que les modules darwiniens ont un domaine spécifique. Selon Cosmides et Tooby, nos esprits consistent largement en « une constellation de mécanismes spécifiques possédant des procédures propres à leur domaine spécifique, qui agissent sur les représentations d'un domaine spécifique, ou les deux » (Cosmides et Tooby, 1994, p. 94). Cela signifie qu'une structure cognitive innée qui est spécifique ne répondra seulement qu'à un input représentationnel d'un type particulier. La cinquième idée, c'est que les modules darwiniens sont des mécanismes computationnels. Comme l'affirment Cosmides et Tooby: 
«notre architecture ressemble à une confédération de centaines de milliers d'ordinateurs fonctionnellement dédiés (souvent appelés modules) [...] » (Tooby et Cosmides, 1995, p. xiii). On doit à Turing l'idée qui sous-tend cette conception. C'est lui qui introduisit pour la première fois l'idée que les processus mentaux sont des computations. De tels dispositifs computationnels sont des ordinateurs classiques. Par conséquent, les processus mentaux cognitifs sont des opérations formelles définies par des représentations mentales structurées syntaxiquement qui sont similaires à des phrases. Une computation est un processus causal qui est conduit syntaxiquement. Finalement, certaines motivations menant à croire à l'existence des modules darwiniens viennent de l'idée de Chomsky selon laquelle l'argument de la pauvreté du stimulus détermine l'information qu'un esprit doit avoir de façon innée. Il faut soustraire l'information provenant de l'environnement de l'information requise à un enfant pour atteindre la maitrise de la langue. Ce qui en résulte est ce à quoi contribuent les connaissances innées de l'enfant à la procédure d'acquisition du langage. Pour les psychologues évolutionnistes, ce qui en résulte et qui est inné est considérable : un esprit qui est largement, mais pas complètement, constitué par des centaines de milliers d'ordinateurs fonctionnellement dédiés. Les modules darwiniens, en somme, qui sont innés, sélectionnés par la nature, fonctionnellement spécifiques, universels, constituent des mécanismes computationnels à la Turing, lesquels, souvent, s'activent de concert avec des ensembles de données spécifiques ou des représentations. Appelons cela "l'Hypothèse de la modularité massive » ou HMM. Cosmides et Tooby ne se sont pas prononcés sur la question de la connexion entre les processeurs computationnels qui ont un domaine spécifique et les ensembles d'informations qui ont des domaines spécifiques. Il semble naturel de joindre les processeurs darwiniens qui ont un domaine spécifique avec les ensembles chomskyens d'information qui ont un domaine spécifique. Appelons cela les modules darwiniens/chomskyens (MDC). J'ai eu de la difficulté à faire ressortir que Cosmides et Tooby n'endossent pas cette position. C'est-à-dire qu'ils pensent qu'il existe des MDC mais qu'il doit bien aussi y avoir d'autres aspects de notre architecture cognitive.

Cosmides et Tooby, en fait, ne s'engagent pas envers la notion selon laquelle l'esprit doit être entièrement modulaire. Pour une première approximation, il faut penser aux possibilités selon quatre choix :

$\begin{array}{ll}\text { Processeurs ayant } & \text { Processeurs ayant } \\ \text { un domaine spécifique } & \text { un domaine général } \\ \text { (Modules darwiniens) } & \end{array}$

Ensemble de données ayant un domaine spécifique

(Modules chomskyens)

Ensemble de données ayant

C

un domaine général 
N.B.: le choix A, par exemple, représente la conjonction de processeurs ayant un domaine spécifique, ou un module darwinien, avec un ensemble de données ayant un domaine spécifique, ou un module chomskyen.

Cosmides et Tooby suggèrent que les choix A, B, C et D sont tous des options viables et que ces quatre options ne sont pas mutuellement exclusives (Cosmides et Tooby, 1994, p. 104). Voici ce qu'ils disent à leur propos: "Chacune de ces possibilités peut être correcte. En effet, toutes peuvent être correctes, bien que dans différents domaines » (Cosmides et Tooby, 1994, p. 104). Ils laissent entrevoir la possibilité que ces options soient vraies, mais dans différents domaines. Pour Cosmides et Tooby, l'esprit n'a pas à être seulement modulaire (ou seulement massivement modulaire). L'esprit, dans leur perspective, peut aussi être non modulaire en certains aspects. Cosmides et Tooby acceptent la représentation massivement modulaire et le modèle processualiste de la cognition qui pose que :

\section{Le principe RMMP}

L'esprit est largement composé d'une vaste assemblée de modules darwiniens/chomskyens. Avertissement : certains choix B, C ou D doivent être des composants de l'esprit.

$\mathrm{Ni}$ Cosmides et Tooby ni moi ne souscrivent à l'idée d'un esprit entièrement modulaire. En même temps, il faut souligner que Cosmides et Tooby souscrivent certainement à la position selon laquelle les modules darwiniens/ chomskyens dominent l'architecture mentale.

Une partie significative du travail de Cosmides et Tooby en psychologie évolutionniste a été dédiée à l'étude du raisonnement humain. En particulier, les effets liés au contenu lors de la tâche de sélection de Wason (1968), ainsi que Wason et Philip Johnson-Laird (1972), ont démontré que l'exécution du raisonnement, quant à différentes tâches qui requièrent l'usage d'une seule règle d'inférence déductive, varie en fonction du contenu en jeu dans les règles d'inférences. Cela viole l'idée la plus fondamentale de la logique formelle, c'està-dire que les arguments sont valides purement en fonction de leur forme abstraite, indépendamment de leur contenu. Que les humains échouent systématiquement à observer l'aspect de la neutralité du contenu quant aux tâches de raisonnements déductifs est apparu comme une surprise considérable. Par exemple, si l'on considère la tâche de sélection de Wason suivante (Johnson-Laird, 1983, p. 30). Un expérimentateur dépose quatre cartes en face d'un sujet avec les symboles suivants:

\section{E $\quad \mathrm{K} \quad 4 \quad 7$}

Le sujet sait que chaque carte possède un chiffre sur un côté et une lettre sur l'autre. L'expérimentateur présente ensuite au sujet les généralisations suivantes: si une carte possède une voyelle sur un côté alors il y a un chiffre pair sur l'autre côté.

La tâche du sujet est de retourner seulement les cartes qui ont besoin d'être observées pour vérifier si la généralisation est vraie ou fausse. L'ordre 
dans lequel les cartes sont retournées n'est pas un problème. Cette tâche apparemment simple s'est révélée être difficile à résoudre. La plupart des gens conçoivent que la carte avec la voyelle doit être retournée. La généralisation demeure valide si, une fois tournée, le chiffre est pair. Si la carte représente un chiffre impair, alors la généralisation doit être fausse. De façon similaire, la plupart des sujets réalisent que la carte avec une consonne ne doit pas être touchée puisque la règle ne dit rien à propos des consonnes. Certains sujets tournent la carte avec le chiffre pair, d'autres non. Mais la carte avec un chiffre pair n'a pas besoin d'être tournée puisque, qu'il y ait une voyelle ou non sur l'autre côté, la conditionnelle sera vraie.

Le problème central concerne la carte avec le chiffre impair. Cette carte doit être tournée puisque, si elle contient une voyelle, alors la généralisation est fausse. Mais peu de sujets insistent sur le fait de la retourner quoique la raison de la vérifier soit exactement identique à la raison poussant à vérifier la carte avec la voyelle : la généralisation est clairement fausse lorsqu'une voyelle et un chiffre impair sont sur les deux côtés de la carte. Wason et Johnson-Laird ont tenté de nombreuses variantes quant à la procédure et au matériel dans le but d'améliorer les performances jusqu'à ce qu'ils trouvent une modification simple ayant des effets frappants (Johnson-Laird, 1983, p. 31). Lorsque les sujets se font présenter quatre cartes représentant des voyages, c.-à-d., avec une destination sur un côté et un mode de transport de l'autre, les résultats se révèlent notablement accrus. Donc, avec les cartes Manchester, Sheffield, Train et Automobile ainsi que la règle générale:

À chaque fois que je vais à Manchester, je voyage en train,

plus de $60 \%$ des sujets comprennent qu'ils doivent retourner la carte indiquant "Automobile ». Cela représente un net contraste avec l'exemple précédent où presque personne n'a compris - dans le cas parallèle où les chiffres impairs devaient être retournés. Et dans le groupe contrôle, à peine plus de $12 \%$ des sujets ont fait le choix équivalent lorsque du matériel abstrait était en jeu. Ce qui suggère que les matériaux réalistes ou familiers produisent de bien meilleurs résultats que les matériaux abstraits ou peu familiers, indépendamment du fait que des expériences distinctes emploient des généralisations avec la même forme logique et les mêmes conditions de vérité. De nombreuses autres variations sur ces expériences ont été effectuées qui semblent confirmer ces résultats.

Toutefois, c'est le travail révolutionnaire de Cosmides et Tooby expliquant les effets liés au contenu lors des tâches de sélection de Wason qui, en partie, a préparé le terrain pour le projet plus général de la psychologie évolutionniste. Cosmides et Tooby expliquaient les effets liés au contenu en invoquant la présence ou l'absence d'un "contrat social » dans la tâche de sélection et non pas la notion associationniste de "familiarité » de Wason et Johnson-Laird. Mais l'idée d'un esprit inné et massivement modulaire se trouvait clairement 
en arrière-plan. La stratégie de « l'ingénierie à rebours » a guidé la méthodologie de ces études. "L'ingénierie à rebours " se rapporte à une stratégie de design expérimental où l'on doit tenter de déterminer les problèmes adaptatifs auxquels les chasseurs-cueilleurs du Pléistocène ont fait face, et, alors, désigner des expériences qui auraient fait ressortir la perspicacité des adaptations fonctionnelles qui sont apparues en réponse à ces problèmes adaptatifs. Il faut comprendre les caractéristiques des conceptions causées par la sélection naturelle afin de clarifier de si complexes adaptations fonctionnelles. Comme le notent Cosmides et Tooby en ce qui concerne le rôle de la chance dans l'évolution:

Des allées et venues aléatoires ne construisent pas systématiquement des arrangements fonctionnels improbables et complexes comme le système visuel, la faculté de langage ou le contrôle moteur. La seule explication connue pour l'existence de conceptions fonctionnelles complexes dans les systèmes organiques est la sélection naturelle (Cosmides et Tooby, 1994, p. 86).

Ce point vaut aussi bien pour la capacité à raisonner chez les humains. En tant que telle, la méthodologie de l'ingénierie à rebours a guidé les études de Cosmides et Tooby sur la tâche de sélection de Wason.

Pour les philosophes, le résultat peut-être le plus stupéfiant de la psychologie empirique des trois dernières décennies a été la déconstruction de la notion qu'il existe une psycho-logique russellienne sous-jacente qui guide l'esprit et le langage. En suivant scrupuleusement les standards de la logique, les humains apparaissent être sauvagement irrationnels dans le sens où il n'y a pas de systèmes logiques employés par les humains qui préservent la vérité, la neutralité quant au contenu et la généralité à travers les domaines d'application. Nous faisons des erreurs inductives et déductives qui sont répandues et influentes. Les résultats expérimentaux soutenant ces affirmations sont robustes et reproductibles. Nous commettons le sophisme de la conjonction, nous sommes coupables de la négligence du taux de base et de l'excès de confiance. Dans les chapitres quatre et cinq, je passe en revue certaines données empiriques concernant ces études. Comme nous l'avons noté auparavant, Cosmides et Tooby ont essayé de renverser les interprétations des théories associationnistes de la disponibilité [associationism-based availability theorists], comme celles de Wason, Kahneman et Tversky, Nisbett et Ross, et d'autres, concernant les effets liés au contenu dans les tâches de sélection de Wason. Les associationnistes argumentent que la familiarité avec les données (ou l'expérience différentielle) explique le fait que les sujets vont raisonner en accord avec, par exemple, le modus tollens dans certains cas, mais violeront le modus tollens dans d'autres. La supposition des associationnistes était que les humains possèdent une capacité de raisonner qui a un domaine général, et indépendante du contenu. Plus tard, les théoriciens du raisonnement pratique, tels que Cheng et Holyoak, ont argumenté, dans le même esprit, en faveur d'une capacité de raisonner qui aurait un domaine général, mais ils suggèrent que les humains emploient des «schémas de raisonnement pratique » qui ont 
été induits par l'expérience récurrente à l'intérieur de domaines ayant des buts définis. Les schémas étaient envisagés comme étant dépendants du contexte, même si les processus cognitifs inductifs engendrant ces schémas étaient pensés comme étant indépendants du contenu. Les expériences différentielles expliquent quels schémas étaient construits et pourquoi certains autres ne l'étaient pas. Toutefois, Cosmides a tenté de montrer que ni les théories associationnistes de la disponibilité ni les théories du raisonnement pratique basées sur l'induction ne peuvent expliquer les effets liés au contenu dans la tâche de sélection de Wason. Elle argumente que la présence d'un contrat social intégré dans la tâche de sélection de Wason explique les effets liés au contenu. S’il existe un contrat social en jeu dans cette tâche, même s'il n'est pas familier aux sujets, alors leurs résultats seront meilleurs. La prédiction de Cosmides a été confirmée par ses expériences. Visiblement, ces résultats empiriques défient la vérité des théories de la disponibilité.

Cheng et Holyoak affirment que les schémas de raisonnements pratiques peuvent expliquer les effets liés au contenu lors de la tâche de sélection de Wason dans le sens où lorsqu'un schéma est actif, les sujets raisonnent en accord avec le calcul des propositions. Inversement, là où aucun schéma n'est visible, les sujets échouent à raisonner en accord avec le calcul des propositions. Cosmides a noté que les règles de contrat social impliquent des règles de permission (ou schémas), mais les règles de permission n'impliquent pas toutes un contrat social. Il en est ainsi à cause de la déclaration du contrat social: "Si le bénéfice revient à un seul, alors il en doit en payer le prix » entraîne la règle de permission "Si quelqu'un entreprend l'action $\mathrm{A}$, alors il doit satisfaire la condition $P$ ». Mais l'inverse ne tient pas. Tous les bénéfices acquis sont des actions qui ont été prises, mais toutes les actions entreprises ne sont pas des bénéfices acquis. Comme Cosmides l'a noté : «Une règle de permission est aussi une règle de contrat social seulement lorsque le sujet interprète "l'action à entreprendre" comme un bénéfice rationné, et la "condition à satisfaire" comme un coût requis " (Cosmides, 1989, p. 237). Il en résulte que le domaine des schémas de permission est plus large que les algorithmes des contrats sociaux.

Ultérieurement, Gigerenzer et Hug ont reproduit les résultats de Cosmides mais ont offert un amendement amical à ces résultats. Ils s'entendent sur le fait que les associationnismes familiers et les hypothèses concernant les schémas de raisonnements pratiques sont faux (ou semblent faux). Mais, ils ont démontré que, lorsque les contrats sociaux qui impliquent des algorithmes de détection de tricheur sont en jeu, les effets liés au contenu apparaissent. En revanche, lorsque seuls les contrats sociaux sont en jeu et aucun algorithme de détection des tricheurs, aucun effet lié au contenu n'est visible. Ensuite, Cosmides et Tooby ont argumenté que les humains pouvaient être de bien meilleurs raisonneurs intuitivement et inductivement qu'ils ne peuvent être de bons raisonneurs intuitivement et déductivement. Une série d'études de Cosmides et Tooby où la négligence du taux de base est en jeu ont montré que si les sujets se font présenter les problèmes comme impliquant des fréquences 
relatives, plutôt que des probabilités appliquées au cas par cas, les résultats sont considérablement meilleurs. Dans le même ordre d'idée, d'autres chercheurs ont obtenu des résultats concordants quant à la fréquence relative appliquée à la négligence du taux de base, le sophisme de la conjonction et le biais de l'excès de confiance. Ces données peignent une image bien plus positive des capacités humaines à inférer inductivement que celle présentée par les théoriciens du raisonnement associationnistes et pragmatiques en 1970 et 1980, ou même par Cosmides et Tooby dans les années 1980. Cependant, le statut du raisonnement déductif reste plutôt faible par rapport aux stricts standards logiques. Avec le rapide survol de la position de Cosmides et Tooby concernant cette partie de la psychologie évolutionniste qui s'occupe du raisonnement humain en question, j'ai essayé de recenser ce que je crois être les implications philosophiques de ces résultats.

Dans le deuxième chapitre, je m'occupe des inquiétudes fondamentales que Jerry Fodor a exprimées contre le projet de la modularité massive. Dans son livre, The Mind Doesn't Work That Way, Fodor argumente que la Nouvelle Synthèse, c.-à-d. la psychologie évolutionniste de Cosmides et Tooby, ou de Pinker et Plotkin, était vraiment mal avisée. Fodor pense que ce qu'il appelle le "problème de l'input ", un problème a priori, immobilise la Nouvelle Synthèse dans son cheminement. Il offre aussi plusieurs objections à celle-ci. Il affirme en particulier que la psychologie évolutionniste ne peut intégrer les inférences globales et abductives dans le contexte de processeurs locaux et computationnels. J'ai soutenu qu'il interprète mal la position de Cosmides et Tooby et charge donc, à maintes reprises, un homme de paille. De plus, j'ai soutenu que l'hypothèse de la modularité massive peut être étendue de façon à expliquer ce que j'appelle «l'abduction évolutionniste non explicite». Évaluer la critique de Fodor était important parce qu'il nous permet de soulever des préoccupations que de nombreux cogniticiens peuvent entretenir à propos de la psychologie évolutionniste et parce que cela nous permettra une compréhension plus fine de la façon dont Cosmides et Tooby conçoivent leur propre projet. Il existe en particulier une rumeur qui circule selon laquelle Cosmides et Tooby souscrivent à la notion d'esprit complètement modulaire. J'espère avoir fait disparaître cette idée complètement erronée. Dans le troisième chapitre, j'ai prospecté des solutions concernant les erreurs de représentation afin de résoudre le problème de la disjonction. La théorie causale rudimentaire de la représentation pose qu'une instanciation de « D » est causée, de façon fiable, par D. Cela établit le fait que les conditions gouvernant ce que signifie pour $\mathrm{D}$ d'être représenté par « $\mathrm{D}$ » est identique à la condition qu'un tel particulier soit vrai. Il est impossible, comme tel, d'introduire la fausseté dans cette perspective. Il est possible de penser que $\mathrm{D}$ cause des instanciations de « $\mathrm{D}$ » est vrai et que $\mathrm{E}$ cause des instanciations de « $\mathrm{D}$ » est faux. Mais cela ne marchera pas. Les « $\mathrm{D}$ » sont causés de manière fiable par les propriétés disjonctives d'être ( $\mathrm{D}$ ou $\mathrm{E}$ ). Comme telles, les instances de E-cause- « D » sont vraies parce qu'elles sont causées de façon fiable par 
(D ou E), et nous n'avons pas de théorie de l'erreur de représentation. Voilà le problème de la disjonction. Je soutiens qu'il est possible de le résoudre en utilisant les ressources de l'hypothèse de la modularité massive de Cosmides et Tooby, combinée avec une contribution étiologique et fiabiliste de la fonction propre des processus computationnels qui constituent de tels modules. Le résultat est une solution aux erreurs de représentations entièrement applicable aux parties de l'esprit qui sont modulaires. Dans les grandes lignes, si le fossé entre le domaine propre (dans lequel le module a été sélectionné) et le domaine actuel (dans lequel nous existons actuellement) est assez large, alors la fonction propre du module faillira. Les erreurs de représentation surviennent quand le fossé entre le domaine propre et le domaine actuel est significatif et résulte en un mauvais fonctionnement des modules. De plus, les types de croyances erronées étaient endémiques chez nos ancêtres si une mauvaise identification des problèmes adaptatifs significatifs en résultait. Les erreurs de représentation sont une partie du processus qui mène au mauvais fonctionnement des modules. Les croyances erronées concernant les problèmes adaptatifs déclenchent des réponses inappropriées des modules. Les erreurs de représentation déclenchent un mauvais fonctionnement, et ce mauvais fonctionnement résulte en une erreur biologique. Inversement, pour nos ancêtres, les types de croyances vraies étaient importants à l'identification des problèmes adaptatifs. Cette contribution ne surestime pas les capacités représentationnelles des modules tout en réussissant à biologiser la contribution de Fodor à la dépendance asymétrique des erreurs de représentations. Je me suis débarrassé de la notion métaphysique d'une "representation consumer" tout en préservant les avantages principaux de la contribution de Millikan quant aux fonctions propres. Cette contribution montre que la signification importe parce que la vérité et la fausseté importent. Dans le chapitre quatre, j'ai soutenu que des représentations lexicales précises ont été cruciales pour la survivance et la reproduction d'homo sapiens sapiens. J'ai soutenu spécifiquement que des processus fiables ont été sélectionnés en raison de leur connexion indirecte, mais étroite, avec les croyances vraies durant la période chasseurcueilleur du Pléistocène de notre histoire ancestrale. Les croyances vraies ne sont pas héréditaires, les processus fiables le sont. Ces processus fiables qui sont connectés avec le raisonnement prennent la forme d'algorithmes darwiniens: une pléthore de règles inférentielles spécialisées et spécifiques à un domaine qui sont constituées pour résoudre des problèmes spécifiques, récurrents et adaptatifs dans les contextes d'échanges sociaux. Les humains, ai-je soutenu, ne raisonnent pas logiquement mais adaptativement. Dans le chapitre cinq, j'ai d'abord souligné que la conception fiabiliste et externaliste de la justification épistémique et de la connaissance fait face à deux groupes de critiques : les épistémologues analytiques et les philosophes des sciences. Le premier groupe reproche aux épistémologues naturalistes de « détourner le sujet » et donc de manquer les débats classiques de l'épistémologie traditionnelle et autres problèmes importants. Le second groupe, ironiquement, reproche aux épistémologues 
naturalistes de "ne pas parvenir à changer de sujet ", à tel point que leurs travaux sont connectés à l'épistémologie analytique traditionnelle, une forme de recherche dépassée et utopique. Les philosophes des sciences pensent que l'épistémologie traditionnelle est utopique parce que c'est une tentative de réponse aux standards déraisonnablement élevés que les sceptiques souhaitent imposer à toute contribution adéquate de la connaissance. Selon les philosophes des sciences, le secret est de rejeter ces standards sceptiques déraisonnablement élevés. Il est clair qu'aucun des deux groupes ne peut avoir raison. J'ai endossé une position en faveur du projet naturaliste en dessinant d'abord une distinction entre les sens mélioratif et non mélioratif de la justification. J'ai utilisé cette distinction pour soutenir que les deux groupes de critiques ont manqué la cible du projet naturaliste mais pour différentes raisons. Ensuite, j'ai passé quelque peu en revue la littérature sur la rationalité humaine du point de vue de la psychologie empirique et suggéré comment une épistémologie naturalisée, qui prend au sérieux ces résultats empiriques et qui est sensible à la distinction mélioratif/non mélioratif, peut être développée. Tout au long de mon cheminement, j'ai essayé de reconnecter l'épistémologie analytique avec la philosophie des sciences en montrant comment les projets naturalistes sont liés dans les deux camps. Parce qu'il me semble qu'il est grand temps que les philosophes reconnaissent que l'épistémologie standard du sujet connaissant est en continuité avec l'épistémologie du scientifique. Quine, selon moi, avait raison : la science est seulement une sophistication du sens commun. Les deux voies de la philosophie analytique, symbolisée par l'analyse informelle mooréenne d'un côté, et l'analyse formelle russellienne de l'autre, ont finalement besoin d'être jointes encore une fois. La façon de le faire, je pense, est de montrer comment une épistémologie naturalisée peut informer et être sensible à une philosophie naturaliste des sciences. Les deux solitudes doivent se fondre l'une dans l'autre. Le chapitre six représente le commencement, à partir des deux chapitres précédents, en ce que cette discussion est directement centrée sur la littérature de la modularité massive et non sur une théorie de la rationalité. Il débute avec une discussion de l'objection, par les non-naturalistes, que la science n'a pas de place dans les problèmes épistémiques. Des non-naturalistes, comme Richard Feldman et Richard Fumerton, ont soutenu que les débats épistémiques sont normatifs ou évaluatifs par nature. Si tel est le cas, il doit être inutile d'employer des ressources factuelles ou descriptives de la science pour reconstruire des notions normatives ou épistémiques comme la justification ou la connaissance. J'ai soutenu que les non-naturalistes font simplement fausse route en cette matière puisque la connaissance est en fait un ensemble de genres naturels qui devraient être étudiés empiriquement, et non un genre conceptuel à étudier par un appel a priori de la raison. J'ai proposé l'argument de la connaissance comme genre naturel (ACGN) pour soutenir ma perspective. En me positionnant de cette façon, je ne me distingue pas seulement du principe central de l'épistémologie analytique, je me distingue aussi de la forme d'analyse de la justification et de la connaissance adoptée dans 
les chapitres quatre et cinq. J'argumenterai pourtant que même sur le terrain ouvert par l'analyse conceptuelle ma perspective est supérieure aux conceptions internalistes de la connaissance et aux autres conceptions externalistes de la connaissance. Par le fait de voir la connaissance comme un ensemble de genres naturels, la mission d'une épistémologie complètement naturalisée, pour la première fois dans l'histoire de l'épistémologie, est-elle finalement offerte ? De ma perspective, il s'agit d'une transformation méthodologique nouvelle et importante qui prépare le terrain à ma contribution modulaire de la connaissance. En somme, ma contribution concernant la connaissance dépend des idées jumelles que la connaissance est un ensemble de genres naturels (et non un genre conceptuel) et qu'une telle connaissance se loge à l'intérieur d'une vaste assemblée de sous-ensembles appropriés de modules RMMP. La connaissance empirique est le résultat des modules darwiniens qui convertissent les inputs sensoriels, si bien que la connaissance a priori est le résultat des modules darwiniens/chomskyens. Une telle connaissance modulaire est instrumentalement importante puisqu'elle résulte, une fois attachée correctement à nos désirs, en la satisfaction de nos besoins biologiques et d'autres buts que nous avons. Il en résulte que la connaissance est composée d'un ensemble de genres naturels logeant dans des modules distincts de l'esprit. Steve Stich a argumenté en faveur du relativisme épistémique dans son classique de 1990, The Fragmentation of Reason. Ce que je crois, c'est qu'il existe une bonne raison de penser que la littérature la plus récente sur la rationalité et la modularité massive se présente à nous avec des assises pour une image différente de la raison et de la représentation. La nouvelle image est, je crois, décidément plus optimiste que celle présentée par Stich. Ma conception modulaire de la connaissance est une espèce de fondationnalisme, bien qu'elle ne conçoive pas la connaissance comme un genre conceptuel mais comme un ensemble de genres naturels. Comme telle, ma perspective de la connaissance et de la justification est incompatible avec le relativisme épistémique, même si elle soutient une fragmentation de la connaissance. Je me distingue d'une autre façon du relativisme de Stich en pensant qu'il existe des assises évolutionnistes pour penser que la vérité a contribué et contribue encore à notre adaptativité en tant qu'espèce. 